\title{
Una experiencia transdisciplinar de trabajo comunitario en contextos de formación profesional
}

\section{A Transdisciplinary Experience of Community Work in Professional Training Contexts}

\author{
Samuel Pérez Norambuena \\ Universidad del Bío-Bío (ubB), Chile \\ sperez@ubiobio.cl \\ ORCID: https://orcid.org/0000-0002-1710-328X \\ Cristóbal Bravo Ferretti \\ Universidad del Bío-Bío (UbB), Chile \\ cbravo@ubiobio.cl \\ ORCID: https://orcid.org/0000000203956046 \\ Marcela Mora Donoso \\ Universidad del Bío-Bío (UBb), Chile \\ mamora@ubiobio.cl \\ ORCID: https://orcid.org/0000-0002-8263-6816 \\ https://doi.org/10.48102/didac.2021..78_JUL-DIC.78
}

8

\begin{abstract}
Resumen
El presente escrito tiene como objetivo comunicar resultados y alcances pedagógicos de una experiencia en docencia universitaria en la formación inicial en la Universidad del Bío-Bío, adscrita a un proyecto de extensión, cuyo propósito fue formar a estudiantes de tercero y cuarto año de las carreras de Pedagogía en Educación Física, Psicología y Trabajo Social, en el ámbito del trabajo comunitario desde una metodología activa. El proyecto se plantea como una respuesta tanto a la estrechez del concepto de formación ciudadana que se desprende del modelo neoliberal que impera en la educación superior como a las limitaciones para un genuino diálogo transdisciplinar en el contexto universitario actual. En esta experiencia se enfatizó en el rol de liderazgo social, desde una perspectiva de trabajo transdisciplinario. Esto permitió fortalecer la ciudadanía activa en las comunidades y el liderazgo social, así como vincularse con la comunidad desde una mirada bidireccional. Desde el enfoque de enseñanza-aprendizaje, fue una experiencia enriquecedora con contextos reales, para resolver problemáticas registradas con los mismos actores sociales.
\end{abstract}

20 - Una experiencia transdisciplinar de trabajo comunitario en contextos de formación profesional Samuel Pérez Norambuena, Cristóbal Bravo Ferretti, Marcela Mora Donoso. DIDAC 78 (2021): 20-31 
Palabras clave: Transdisciplinariedad; educación superior; aprendizaje y servicio; ciudadanía activa; trabajo comunitario.

\begin{abstract}
The present document aims to communicate the results and pedagogical scope of an experience in university teaching initial training at the University of Bio-Bio, assigned to an extension project, whose objective was to train third and fourth year students of Pedagogy in Physical Education, Psychology and Social Work, in the field of community work from an active methodology. The project is proposed as a response to the narrowness of the concept of citizenship training that emerges from the neoliberal model that prevails in higher education and to the limitations for a genuine transdisciplinary dialogue in the current university context. In this experience, emphasis was placed on the role of social leadership, from a transdisciplinary work perspective. This made it possible to strengthen both active citizenship in the communities and social leadership, as well as to create a link with the community from a two-way perspective. From the teaching-learning approach, it was an enriching experience with real contexts, to solve problems raised with the same social actors.
\end{abstract}

Keywords: Transdisciplinarity; Higher Education; Learning and Service; Active Citizenship; Community Work.

Fecha de recepción: 19/01/2021

Fecha de aceptación: 14/03/2021

\section{Introducción}

En el contexto universitario actual, cobra relevancia el compromiso social de los estudiantes y su reconocimiento como agentes de cambio. Tal como señala el Programa de las Naciones Unidas (2021), en su cuarto objetivo de desarrollo sostenible —la educación de calidad-, es necesario "facultar a los educandos para que sean ciudadanos del mundo, creativos y responsables". No obstante, las políticas educacionales neoliberales que se han implementado desde hace unas cuatro décadas en el contexto latinoamericano, como la de "transformar las estructuras y la organización de los sistemas educativos, mediante la lógica de la libre competencia del mercado" (Lopez \& Flores, 2006, p. 3) suponen importantes obstáculos para una formación que se encamine en dicha dirección. Prueba de ello son las reformas vivenciadas por las universidades a partir de Bolonia que tendieron a homogeneizar los procesos formativos (Bonal, Tarabini-Castellani \& Verger, 2007; Dale, 2007; Green, 2007; Santos, 2005). Una de las implicaciones fue la globalización a nivel educativo que relevó los procesos de mercantilización de la educación superior, lo cual evidenció la estrecha vinculación de los programas de formación con las necesidades del mercado laboral (Espinoza, 2014, p. 149).
Lo anterior es un factor que tiene como resultados la fragmentación de las áreas del conocimiento, la estrecha interpretación del diálogo disciplinar y una formación ciudadana débil como características del actual modelo de formación universitaria; situaciones que dificultan el surgimiento de interés y el involucramiento activo de estudiantes en temas relacionados con el bienestar social y el desarrollo comunitario (Friz Echeverría, 2018).

Por otra parte, Leite y Genro (2012, p. 764), mencionan la concepción de una nueva epistemología de la educación superior: "la cual surge en el siglo Xxi. Esta epistemología sostiene las razones por las que las universidades deben trillar caminos globales e internacionales, rediseñar su perfil en dirección a los mercados y desarrollar modalidades de capitalismo académico".

A partir de estas críticas en la formación de pregrado, se comparte la sistematización de una experiencia en docencia que expone y analiza los alcances pedagógicos de una innovación en la formación de pregrado, en una universidad pública y estatal de Chile, la cual consiste en la implementación de un conjunto de intervenciones llevadas a cabo por equipos de estudiantes de distintas carreras en conjunto con agrupaciones comunitarias del entorno de los

Una experiencia transdisciplinar de trabajo comunitario en contextos de formación profesional $\bullet 21$ Samuel Pérez Norambuena, Cristóbal Bravo Ferretti, Marcela Mora Donoso. DIDAC 78 (2021): 20-31 
campus académicos de la Universidad del Bío-Bío en la ciudad de Chillán (Fernando May y La Castilla). La experiencia que da lugar a este artículo proviene de un proyecto de extensión académica titulado Estimulando el Trabajo Comunitario desde la UвB: Formación e Intervención en Comunidades Locales, desarrollado en 2018. De este modo, orientándose por los principios de la Metodología Aprendizaje y Servicio, se propuso realizar una experiencia formativa que buscó desarrollar conciencia en los estudiantes acerca de su respectivo rol de liderazgo social mediante estrategias de trabajo transdisciplinario en el ámbito comunitario, apuntando al diálogo entre los distintos saberes, incluidos aquellos que surgen al interior de las propias comunidades, dando respuesta así a las falencias formativas por la excesiva especialización y fordismo de los planes de estudio que no procuran la convergencia entre saberes, ni mucho menos entre carreras que alberga una misma facultad.

Ciudadania y tensiones en la formación universitaria El ámbito de la ciudadanía ha entrado en una situación de crisis en las sociedades contemporáneas. Diversos autores han apuntado al individualismo, la fragmentación social y la despolitización como algunas de las categorías que en la actualidad rigen la organización de la sociedad y que han socavado profundamente la noción de ciudadanía (Escobar, 2006).

En América Latina el concepto de ciudadanía ha estado particularmente marcado por las interrupciones de los regímenes democráticos y por las secuelas traumáticas de las dictaduras que se impusieron en la región durante el último cuarto del siglo xx. Si bien las últimas décadas han supuesto importantes avances en la democracia electoral y el reconocimiento y el ejercicio de derechos políticos, en la actualidad persiste una "ciudadanía de baja intensidad" (PNUD, 2004, p. 145) que no necesariamente ha implicado el desarrollo de otros derechos de participación social.

Desde un punto de vista histórico es posible señalar que en el ámbito social y político ha existido una marcada tendencia en Chile a conceptualizar la ciudadanía de manera estrecha, como si fuera una concesión otorgada por el Estado a la sociedad y que se materializa en el ámbito jurídico e institucional (Salazar, 2006).

El desarrollo de la ideología neoliberal ha favorecido que el consumo emerja como uno de los principales referentes de identidad y que, mediante el acceso a los bienes que provee el mercado, se experimente un efecto de integración social. Esta lógica de funcionamiento ha impregnado en otros planos de participación, lo que se aprecia en el hecho de que las demandas por las que se moviliza la sociedad civil son particulares, inmediatas y locales (Escobar, 2006).

En el contexto educativo chileno, desde la recuperación de la democracia se han puesto en marcha esfuerzos graduales para fortalecer la ciudadanía a través de distintos instrumentos como la reforma educacional y la ley 20.911 de 2016, que establece un Plan de Formación Ciudadana desde la Educación Pública Preescolar (Mineduc, 2018). A pesar de esto, ha existido una gran dificultad para lograr cambios sustantivos. Estudios internacionales señalan que Chile, a nivel escolar, tiene un puntaje significativamente menor a la media mundial en formación ciudadana (Schulz et al., 2016).

De acuerdo con Muñoz et al. (2019), la formación ciudadana en el sistema educacional enfrenta en la actualidad los siguientes desafíos:

1. Hacer de la formación ciudadana una tarea explícita de los contextos educativos.

2. Acercar la formación ciudadana a los intereses de los estudiantes.

3. Superar el vínculo con la dimensión electoral de la política.

4. Potenciar los análisis colectivos por sobre los individuales.

5. Ampliar el concepto de ciudadano: del ciudadano elector al ciudadano como actor social.

En el caso de la universidad, existen factores estructurales derivados del modelo de educación neoliberal

22 - Una experiencia transdisciplinar de trabajo comunitario en contextos de formación profesional Samuel Pérez Norambuena, Cristóbal Bravo Ferretti, Marcela Mora Donoso. DIDAC 78 (2021): 20-31 
implementado en las últimas cuatro décadas que pueden explicar la dificultad para conseguir que la ciudadanía activa sea parte de la formación integral a la que se aspira. Una de las consecuencias de las políticas neoliberales en la educación superior ha sido la transformación de la misión universitaria, la cual ha asumido una orientación más hacia el entrenamiento profesional que hacia la transformación social (Mollis, 2010).

Por otra parte, en este contexto de reformas, se instaló en la educación superior una cultura de la medición influenciada principalmente por las agencias internacionales de financiamiento como el Banco Mundial o el Fondo Monetario Internacional. Este modelo fue el que, según Iriarte y Ferrazzino (2013, p. 3), "incorporó en el análisis de los sistemas educativos de nivel superior el discurso de la calidad y la eficiencia, así como el tema de la evaluación según los criterios de productividad".

Un tercer factor apunta a la restricción del gasto fiscal y el crecimiento del sector privado en materia de educación superior, lo que se ha traducido en modelos educativos que se orientan más hacia la satisfacción de demandas del mercado que a los intereses públicos y sociales del país (Espinoza, 2016).

Entre las pautas implementadas en la región para crear un mercado educativo están principalmente las relacionadas con el recorte al presupuesto universitario (Castelao-Huerta, 2021). En Brasil, el gobierno ha decidido abandonar las instituciones públicas de educación superior a su propio destino; se ha ido desmantelando del aparato científico-tecnológico y predomina una mirada empresarial de la educación superior (Borges \& Fernández Aquino, 2012). De igual manera, en México, desde la década de 1990 persiste una reducción de financiamiento a las universidades públicas (Aboites, 2012). En los casos colombiano y chileno se ha optado sistemáticamente por financiar las universidades privadas en desmedro de las instituciones de carácter público (Ramos \& Parra, 2017; Oyarce, Leihy \& Zegers, 2020). Estas consecuencias en la educación superior vienen aparejadas de individualismo académico, preocupación por el mercado laboral, deterioro de un trabajo colaborativo y enfoques curriculares por competencias.

Como señalan Iriarte y Ferrazzino (2013, p. 11), la educación superior en América Latina se debe orientar hacia dos metas:

una es transmitir a los estudiantes las capacidades
necesarias para enfrentar la multiplicidad de acti-
vidades que el mundo profesional demanda; otra,
es la que apunta a generar y extender los princi-
pios que hacen a la ciudadanía favoreciendo de
este modo a la inclusión social. Finalmente, am-
bos caminos se entrecruzan y fortalecen.

Si bien la formación ciudadana es un objetivo explícito en la mayoría de los programas y las políticas de las instituciones de educación superior, las políticas neoliberales en el ámbito educativo han operado desde un concepto despolitizado de ciudadanía que preconiza la integración competitiva de los jóvenes profesionales en el mercado laboral, por sobre el rol que cumplen en los procesos de transformación social. Como plantea Friz Echeverría (2018, p. 169): "la educación ciudadana no logra desembarazarse de la impronta economicista neoliberal que asemeja, o tiende a identificar incluso, al ciudadano con el agente económico, ya en su forma de oferente, productor, demandante o consumidor".

La formación ciudadana activa requiere trascender la mera declaración formal y el traspaso de conocimientos procedimentales, muchas veces descontextualizados, para pasar al desarrollo de un conjunto de actitudes, habilidades y valores que apunten al reconocimiento del otro y al empoderamiento de los estudiantes como protagonistas de cambios sociales.

Concordamos con Muñoz et al. (2019, p. 22) cuando plantean que:

Dentro de las habilidades, la formación ciudadana debería promover aquellas vinculadas a la formación de un juicio crítico e independiente, el diálogo, el respeto y la colaboración con otros. También, aquellas habilidades que propician la 
participación, el debate, el reconocimiento de los otros como legítimos otros, así como aquellas que contribuyen a que el sujeto posea una mayor apertura al mundo y a la realidad, siendo capaz de enfrentar y resolver problemas y conflictos de manera pacífica.

\section{Formación universitaria y la necesidad de lo transdisciplinar}

La universidad y su función como espacio de generación y transmisión del conocimiento ha sido el lugar que, por excelencia, ha favorecido y sostenido la mirada del mundo y de los fenómenos desde lo disciplinar. De este modo, se ha promovido el conocimiento parcial, lineal y lógico de la realidad. Para esto se ha desarrollado una organización del conocimiento en disciplinas altamente especializadas y fragmentadas, pero que de acuerdo con Martínez (2003) resultan menos adecuadas para tratar los problemas intelectuales y sociales más importantes de nuestro tiempo.

Tanto el informe oCDE (2009) como Oliva et al. (2018) indican que en la educación superior chilena la flexibilidad limitada y la sobreespecialización del currículum representan deficiencias estructurales genéricas del sistema. Esta estructura hace que la formación sea altamente especializada, sin posibilidades de acceso a espacios complementarios, en la que predominan ciertas formas y lógicas de organización disciplinar sobre otras. Al respecto, Nicolescu (1996) indica que las miradas profundas de las disciplinas son importantes y necesarias, pero al mismo tiempo insuficientes de cara a la comprensión de la realidad que ha dejado de ser mecánica y que ya no puede ser entendida de manera disciplinar.

Este modelo formativo predominante, al igual que el económico, social y político, está siendo cuestionado e invitado, lentamente, a conectarse con la sociedad y sus necesidades, como "eliminar la pobreza, la intolerancia, la violencia, el analfabetismo, el hambre, el deterioro del medio ambiente y las enfermedades, y a las encaminadas al fomento de la paz, mediante un planteamiento interdisciplinario y transdisciplinario" (Unesco, 1996, s. p.).
Bajo este contexto de crisis, resulta necesario que las universidades responsablemente se orienten a mirar los fenómenos sociales como un entramado complejo, amplio y divergente. Este desafío requiere de esfuerzos para desarrollar una concepción integradora del conocimiento. Lo transdisciplinar logra esta integración, ya que permite la experimentación, la generación de proyectos que aúnen miradas y que se encarnan en prácticas de transformación local y regional; asimismo, es la puerta abierta que queda para que las universidades se dejen influir por lo social. Tal como lo plantea Morín (2003, p. 29): "vivimos bajo el imperio de los principios de disyunción, de reducción y abstracción, cuyo conjunto constituye el paradigma de la simplificación", lo que potencia el pensamiento reduccionista, en circunstancias en las que cada componente es parte de un todo y es esta realidad global la que se debe proyectar desde la formación universitaria.

\section{Enfoques formativos universitarios con mirada critica y social: aprendizaje y servicio}

Los enfoques formativos universitarios están transitando desde estrategias centradas en el contenido hacia aquellas centradas en el aprendizaje. De este modo, la metodología aprendizaje y servicio se presenta como una alternativa que permite importantes ventajas para el trabajo con comunidades, pues fortalece la formación de la ciudadanía en el contexto universitario e imbrica los saberes disciplinarios y las habilidades transversales.

La metodología activa aprendizaje y servicio tiene como particularidad enfatizar en las intencionalidades pedagógicas de los contenidos disciplinares y transversales o genéricos; así un estudiante no sólo aprenderá su disciplina, sino que también desarrollará otras habilidades, sobre todo las competencias sociales, que permitirán generar un pensamiento más complejo para observar la realidad. Su foco es la experiencia in situ con la comunidad, "caracterizada por integrar en el currículum académico, actividades de servicio con y para la comunidad" (Quintana et al., 2018, p. 94).

Según Furco et al. (2002), la metodología aprendizaje y servicio está sustentada en tres ejes: 1) en el

24. Una experiencia transdisciplinar de trabajo comunitario en contextos de formación profesional

Samuel Pérez Norambuena, Cristóbal Bravo Ferretti, Marcela Mora Donoso. DIDAC 78 (2021): 20-31 
marco de una asignatura para la resolución de un problema en comunidad; 2) como movimiento pedagógico que enfatiza la formación ciudadana y la solidaridad, en contexto de dinámicas nacionales, crisis, desastres naturales o problemáticas sociales (Cecchi, 2006; Tapia, 2010); y 3) como trabajo in situ para potenciar habilidades tanto disciplinarias como genéricas en los estudiantes.

Esta experiencia educativa se sustentó con los aportes de la Universidad de Stanford, desde el punto de vista del modelo de Aprendizaje y Servicio (Service Learning 2000 Center, 1996) con base en los cuadrantes del aprendizaje y servicio, lo que permite generar una vinculación real y bidireccional entre la academia-universidad y comunidad. En este contexto comunitario, los estudiantes movilizan conocimiento disciplinar, conceptual, procedimental y actitudinal para concretar una actividad solidaria en conjunto con la comunidad, que permita solucionar problemáticas de la población (Briede \& Mora, 2016), lo que contribuye al desarrollo regional y a proyectarnos como institución pública y estatal hacia y con la comunidad.

Por otro lado, los aportes epistémicos que subyacen a la metodología aprendizaje y servicio se encuentran en los referentes de la pedagogía crítica latinoamericana. Tal como plantea Freire (1989), el conocimiento adquiere características de ser fuente de liberación y los supuestos teóricos que lo acompañan son: participación social, comunicación horizontal entre los actores que integran los estamentos, significación de imaginarios simbólicos, humanización de los procesos educativos, contextualización del proceso educativo y transformación de la realidad social.

Por esto, resulta crucial instaurar acciones pedagógicas en una institución de educación, basadas en una pedagogía crítica regida por prácticas cooperativas, reconocidas como espacios de acción social, donde cada miembro asuma responsabilidades específicas de gestión y consolidación de procesos autónomos (Aguirre, 2003).

En consecuencia, la metodología aprendizaje y servicio considera estas características que estable- cen los autores, las cuales deben implementarse en la formación universitaria y aún más allá, con el sentido de la transdisciplinariedad entre carreras.

De esta manera, la metodología se implementó considerando las siguientes fases:

1. Formular objetivos y habilidades a proyectar en la formación universitaria, es decir, repensar el currículum formativo para dar paso a cómo organizar las acciones curriculares.

2. Definir actividades que realizará el estudiante y el docente con y en la comunidad, que permitan ser significativas para la formación universitaria.

3. Determinar cómo evidenciará ese aprendizaje en respuesta de un problema real de la población. (Quintana et al., 2018, p. 95).

\section{Experiencia de formación ciudadana transdisciplinar} con enfoque de aprendizaje y servicio

Nuestra mirada transdisciplinaria de formación de profesionales tiene como principios lo diverso, la formación integral y la construcción de conocimientos de manera colectiva. Formamos al estudiante para que sea un ciudadano y profesional con capacidad crítica, reflexiva y creativa, capaz de desenvolverse y vincularse en y con los diferentes contextos sociales y sus particularidades.

Esta experiencia tiene su origen en algunos factores educativos que quisimos enfrentar de una manera diferente: la poca relación en la formación con otras disciplinas; la escasa vinculación entre académicos de distintas carreras en temas fuera de lo disciplinario $\mathrm{y}$, por último, una vinculación con las comunidades muy acotadas en términos de su especificidad. Estas tensiones constituyen problemáticas que es necesario transformar, por lo que se incluyeron académicos de las carreras de Pedagogía en Educación Física, Psicología y Trabajo Social, carreras pertenecientes a la Facultad de Educación y Humanidades. Estos académicos, a través de las asignaturas de Talleres Extraescolares, Psicología Comunitaria e Intervención Social Territorial y Comunitaria, respectivamente, se relacionaron para llevar a cabo esta experiencia forma- 
tiva. Conjuntamente participaron 21 estudiantes, siete de cada especialidad que cursaban el tercer y cuarto año de sus carreras. El proyecto involucró a siete comunidades locales de base territorial, en su mayoría compuestas por juntas de vecinos, cuyos integrantes se reúnen periódicamente en torno a la gestión de necesidades y metas de interés barrial. Entre éstas se encuentran: el cuidado del entorno, el desarrollo de actividades recreativas, temas asociados a la vejez y a la salud mental. Las agrupaciones participaron de manera voluntaria, acompañadas por tríos de estudiantes (uno por carrera), que de manera transdisciplinar debieron responder a los desafíos de esas comunidades.
Desde lo pedagógico se procuró la mirada transdisciplinar mediante la metodología aprendizaje y servicio, dado que, a partir de una actividad curricular los estudiantes movilizan saberes disciplinarios para aportar a la comunidad y contribuir en su desarrollo, considerando las inequidades existentes en la sociedad neoliberal.

Con el fin de sistematizar la experiencia formativa, se presentan en la Tabla 1 las fases de acuerdo con la metodología activa aprendizaje y servicio, y se exponen las actuaciones que realizaron los estudiantes desde una mirada transdisciplinar entre carreras.

Tabla 1

Fases de la metodología desarrollada

\begin{tabular}{|c|c|c|c|}
\hline FASES DEL PROYECTO & DESCRIPCIÓN DE LA ACTIVIDAD & $\begin{array}{l}\text { TRABAJO COLABORATIVO Y CON LA } \\
\text { COMUNIDAD }\end{array}$ & Resultados DE LA FASE \\
\hline \multirow[t]{3}{*}{ Diagnóstico } & $\begin{array}{l}\text { Se realizaron encuentros académicos } \\
\text { entre docentes de las distintas } \\
\text { carreras para abordar la formación } \\
\text { práctica con criterios } \\
\text { multidisciplinares. }\end{array}$ & $\begin{array}{l}\text { De manera colaborativa, entre } \\
\text { los académicos se dialoga sobre los } \\
\text { criterios de participación de los } \\
\text { principales agentes: académicos, } \\
\text { estudiantes y agrupaciones. Asimismo, } \\
\text { se conversa sobre las necesidades } \\
\text { formativas de los estudiantes para el } \\
\text { proyecto. }\end{array}$ & $\begin{array}{l}\text { Se definieron los criterios de } \\
\text { participación: voluntaria, agrupaciones } \\
\text { consolidadas cercanas a los campus de } \\
\text { la universidad y estudiantes que } \\
\text { estuvieran en tercer ańo y cursando } \\
\text { alguna práctica. El número de } \\
\text { participantes fue con base en criterios } \\
\text { de sostenibilidad. }\end{array}$ \\
\hline & & & $\begin{array}{l}\text { Se estableció vincular los objetivos del } \\
\text { proyecto con las mallas curriculares de } \\
\text { cada carrera. }\end{array}$ \\
\hline & $\begin{array}{l}\text { Esta fase buscaba dos grandes hitos: } \\
\text { catastro de los estudiantes por } \\
\text { carrera y de agrupaciones } \\
\text { interesadas. }\end{array}$ & $\begin{array}{l}\text { Los académicos llevaron a cabo un } \\
\text { trabajo en terreno, realizando difusión } \\
\text { del proyecto e identificando líderes y } \\
\text { representantes de las agrupaciones. }\end{array}$ & $\begin{array}{l}\text { Se eligieron siete agrupaciones y siete } \\
\text { estudiantes bajo los criterios } \\
\text { establecidos. }\end{array}$ \\
\hline $\begin{array}{l}\text { ACERCAMIENTO A LOS } \\
\text { PARTICIPANTES }\end{array}$ & & $\begin{array}{l}\text { Además, en las clases de estos } \\
\text { académicos se informa del proyecto a } \\
\text { estudiantes y se les invita a participar. }\end{array}$ & \\
\hline
\end{tabular}

\begin{tabular}{|c|c|c|c|}
\hline \multirow{3}{*}{$\begin{array}{l}\text { FORMACIÓN EN } \\
\text { TRABAJO } \\
\text { COMUNITARIO }\end{array}$} & $\begin{array}{l}\text { Se plantea un proceso formativo que } \\
\text { permita nivelar conocimientos } \\
\text { básicos para el trabajo comunitario. }\end{array}$ & $\begin{array}{l}\text { De manera previa al trabajo con la } \\
\text { comunidad, los estudiantes } \\
\text { participaron en los talleres de } \\
\text { aprendizajes activos, lo cual los llevó } \\
\text { a conocerse y a construir un relato } \\
\text { colectivo sobre la intervención futura. }\end{array}$ & $\begin{array}{l}\text { Se realizó una formación obligatoria } \\
\text { destinada a los estudiantes con } \\
\text { contenidos en temas comunitarios, } \\
\text { territorio, ética de la intervención, } \\
\text { liderazgo, trabajo en equipo y } \\
\text { habilidades comunicativas, entre otros. }\end{array}$ \\
\hline & & & $\begin{array}{l}\text { Se estableció un contrato de } \\
\text { compromiso y responsabilidad social } \\
\text { con las comunidades. }\end{array}$ \\
\hline & & & $\begin{array}{l}\text { Se formaron los equipos de trabajo } \\
\text { compuestos por un estudiante de cada } \\
\text { carrera. }\end{array}$ \\
\hline
\end{tabular}

26 - Una experiencia transdisciplinar de trabajo comunitario en contextos de formación profesional Samuel Pérez Norambuena, Cristóbal Bravo Ferretti, Marcela Mora Donoso. DIDAC 78 (2021): 20-31 
Diagnóstico DE LAS AGRUPACIONES
Cada grupo tomó contacto con una agrupación comunitaria asignada. Aplicaron instrumentos (entrevistas, encuestas y talleres) para detectar necesidades.
Los equipos se presentan en las comunidades, dan a conocer los motivos de este acercamiento y coordinan algunos pasos de la intervención.
Cada equipo (triada) de manera colaborativa levantó información de la comunidad a partir de la aplicación de instrumentos elaborados en conjunto con académicos. Se indaga en: datos sociodemográficos, socioculturales, salud, principales problemáticas, necesidades, recursos y fortalezas de la comunidad.

\begin{tabular}{l}
\hline \\
IMPLEMENTACIÓN DE \\
LA INTERVENCIÓN \\
COMUNITARIA
\end{tabular}

Se le asignó un académico supervisor a cada grupo para acompañar el proceso de trabajo con la comunidad.
Se generaron procesos de coconstrucción de estrategias de resolución para las necesidades y problemáticas levantadas.

Planificación y ejecución de cinco sesiones de trabajo con apoyo de académico. Esto fue abordado de manera transdisciplinar, por lo que cada estudiante aportaba desde su disciplina de modo colaborativo. Algunos de los talleres realizados fueron: gestión de espacio para la organización social de la comunidad, liderazgo, comunicación, proyectos, entre otros.

Se plantean distintas acciones cuya finalidad es evaluar el trabajo realizado y sus alcances. Éstas fueron construidas entre los participantes.
FASE DE EVALUACióN DE LA EXPERIENCIA FORMATIVA
Seminario reflexivo de experiencias comunitarias con enfoque

transdisciplinar: cada grupo de participantes realizó una presentación de su experiencia.

Retroalimentación durante todo el proceso con tutores, triadas asignadas y con la comunidad.

Bitácoras de la intervención.

Encuentro reflexivo final. Se formó un grupo focal con un grupo representativo de estudiantes.
Se detectaron distintos elementos del proyecto que se resumen de la siguiente manera:

\section{Fortalezas}

"Las tres carreras trabajan con el elemento humano, pero la forma de pensar es muy diferente", estudiante de EF.

"El elemento más importante fue la confianza entre compañeros y como [sic] una buena comunicación es fundamental para construirla", estudiante de Psicología.

"Fortaleció los lazos de la comunidad en base a la comunicación y ayuda de los vecinos", vecina VFM.

"Existía un liderazgo compartido entre los compañeros, que se daba de manera natural", estudiante de Psicología.

Debilidades

"Es complicado reunirse de manera sistemática con los estudiantes porque ellos tienen clase y otros compromisos", académico de Educación Física.

"Nos costaba organizarnos como grupo de acuerdo con las necesidades detectadas por trabajos de la universidad", estudiante de Trabajo Social.

Oportunidades

"La buena disposición de las comunidades para recibir nuevas ideas y proyectos", académico de Psicología.

Amenazas

"La disponibilidad y los horarios de las comunidades fueron un tema, ya que ellos trabajan o tienen poco tiempo", estudiante de Psicología.

"La comunidad no trabajaba por un mismo fin, sino por pasajes y eso generó mucha dificultad", estudiante de Psicología.

Fuente: Elaboración propia 
Para finalizar este apartado, presentamos una gráfica que resume los distintos elementos de la fase metodológica y sus relaciones, que fueron desarrollados en la experiencia descrita.

Gráfica 1

Metodología de formación transdisciplinar para el trabajo comunitario

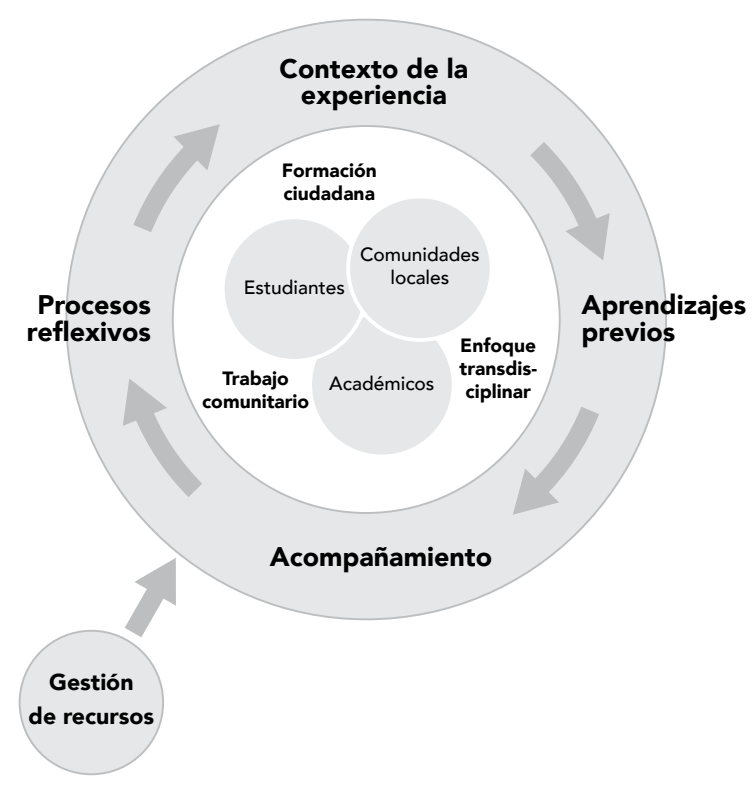

Uno de los pasos formativos importantes en esta experiencia fue procurar el proceso reflexivo en la formación, las implicancias éticas de las labores y actividades curriculares realizadas en, con y para la comunidad. Esto permitió que mediante las técnicas de grupos focales los estudiantes pudieran expresar a través de sus discursos los aportes que la experiencia significó para ellos, planteada desde tres carreras, otorgando la transdisciplinariedad, y los aprendizajes que en ella se vivieron. A manera de ejemplo se eligieron algunas citas textuales de estudiantes que permiten visibilizar que al estar vinculado en terreno y con problemáticas reales, la formación se humaniza, se fortalece y, más aún, se mira desde la complejidad con los aportes de los demás compañeros de otras carreras.

\section{Conclusiones}

La experiencia llevada a cabo tuvo como propósito acercarse a las comunidades locales y a sus intereses más allá de una mirada parcelada y desconectada de otras fuentes de conocimiento. Esto trajo una serie de desafíos a los distintos participantes, quienes debieron responder a éstos de manera colectiva. Es decir, nuestra primera gran conclusión, en consonancia con lo expuesto por Skewes (2013), es que para desarrollar un trabajo con el enfoque transdisciplinar es necesario y pertinente reconocer que éste se da a través de la experimentación y el diálogo permanente entre los diferentes actores, durante todo el proceso, lo que permite ligar miradas e interpretaciones de los sujetos sociales. Tal proceso contribuye a la familiarización con la comunidad y al desarrollo gradual de estrategias de trabajo que tiendan a la horizontalidad. En este sentido, un aprendizaje fundamental que se recoge de la experiencia es el de reconocer y validar los recursos y potenciales de la comunidad para lograr cambios y gestionar sus necesidades.

Otro elemento que vale la pena mencionar es la relevancia de que la educación de los futuros profesionales sea diseñada en espacios formativos y de intervención abiertos, junto a otras disciplinas. Dar este paso implica superar el asignaturismo y el conocimiento parcelado de las aulas y generar herramientas para el trabajo comunitario en espacios diversos e incluyentes que permitan enriquecer y complementar el abordaje de la realidad social y la creación de relatos colectivos para dicho compromiso. El diálogo disciplinar debe ser potencialmente transformador del conjunto de conocimientos y habilidades disciplinares, tal como el estudiante las ha interiorizado en su proceso formativo. Para esto es necesario fomentar la actitud de apertura y de valoración del encuentro de saberes como fuente de recursos creativos para poner en práctica en el trabajo comunitario. Sin duda, la transdisciplinariedad en el marco del trabajo comunitario debe aspirar a modificar los propios esquemas de referencia disciplinar con que operan los profesionales de las distintas especialidades.

Para que se produzcan proyectos de aprendizaje y servicio desde lo transdisciplinar, a partir de esta experiencia deducimos que resulta imprescindible la presencia de académicos que estén muy comprometidos con esta metodología y con el proceso desde el

28 - Una experiencia transdisciplinar de trabajo comunitario en contextos de formación profesional Samuel Pérez Norambuena, Cristóbal Bravo Ferretti, Marcela Mora Donoso. DIDAC 78 (2021): 20-31 
inicio hasta el final. Son ellos lo que motivan, refuerzan, acompañan y coconstruyen junto a los estudiantes y las comunidades el diálogo y el abordaje más allá de lo disciplinar. Sin embargo, desde la experiencia llevada a cabo, creemos que esto requiere de políticas universitarias que tiendan a profundizar la formación ciudadana, promuevan el diálogo y la práctica conjunta de distintas áreas de formación.

La evaluación constante del proceso es un hecho que caracterizó esta experiencia. Se considera pertinente que este tipo de abordaje incluya varios momentos de monitoreo entre distintos actores; de reflexión y retroalimentación que enriquezcan la relación que propician los equipos profesionales con las comunidades. Este proceso de diálogo puede ir contribuyendo a que los estudiantes en práctica vayan desprendiéndose del rol de experto y de miradas paternalistas de las intervenciones, que han prevalecido en la formación profesional (Atria, 2012). En este sentido, se destaca el valor de la experiencia realizada para entender el trabajo comunitario como un proceso que contempla la evaluación-reevaluación de las estrategias de intervención en función de las características del grupo y se relevan los procesos de reflexión al interior de los equipos de trabajo transdisciplinarios. Esto es una parte importante de la implementación de la metodología aprendizaje y servicio, que permite considerar las implicancias éti- cas, así como los resultados con relación al trabajo en contexto.

Es fundamental demostrar una valoración de los grupos con que se trabaja, en sus maneras de congregarse y construir sus historias. La experiencia vivida permite trascender la comprensión instrumental de la participación social para entenderla como un fin en sí mismo. Avanzar en la dirección de la participación tiene efectos empoderadores, en tanto las comunidades comienzan a experimentar transformaciones individuales y sociales que las posicionan mejor para enfrentar los cambios que implica la búsqueda de su bienestar social (Montero, 2004).

El trabajo comunitario puede ser entendido como un proceso abierto y en curso, en el que no hay recetas en cuanto a "la" forma de intervención. La experiencia permitió distinguir un escenario ideal de un escenario real de intervención, reconociendo la distancia entre las descripciones teóricas y academicistas de lo comunitario, de práctica real en comunidades locales con sus propias tensiones, problemáticas y aspiraciones.

Por último, aquí hemos expuesto los desafíos de la formación inicial docente y la formación inicial de otras carreras, y ya que requieren del trabajo con profesionales diversos para abordar una problemática de contexto, entonces resulta esencial que la universidad visualice estas reformas en la docencia.

REFERENCIAS

Aboites, H. (2012). El derecho a la educación en México: del liberalismo decimonónico al neoliberalismo del siglo xxI. Revista Mexicana de Investigación Educativa, 17(53), 361-389.

Aguirre, L. (2003). El sentido de la pedagogía crítica en la era de la globalización después del 11 de septiembre de 2001: Entrevista a Peter McLaren. Revista Electrónica de Investigación Educativa, 5(1), 1-51.

Atria, F. (2012). La mala educación. Ideas que inspiran al movimiento estudiantil en Chile. Santiago: Catalonia-Ciper.

Bonal, X., Tarabini-Castellani, A. \& Verger, A. (2007). La educación en los tiempos de globalización: Nuevas preguntas para las ciencias de la educación. En X. Bonal, A. TarabiniCastellani \& A. Verger (Comps.), Educación y globalización. Textos fundamentales (pp. 13-35). Buenos Aires: Miño y Dávila.

Borges, M. \& Fernández Aquino, O. (2012) Educação Superior no Brasil e as políticas de expansão de vagas do Reuni: avanços e controvérsias. Educação Teoria e Prática, 22, 117 138.

Briede, J. C. \& Mora, M. (2016). Diseño y co-creación mediante aprendizaje y servicio en contexto vulnerable: análisis de percepción de la experiencia. Formación Universitaria, 9(1), 57-70. Recuperado de https://dx.doi.org/10.4067/S0718-50062016000100007

Castelao-Huerta, I. (2021). Investigaciones sobre los efectos de la neoliberalización de la educación superior pública en América Latina 1. Educação e Pesquisa, 47. Recuperado de https://dx.doi.org/10.1590/s1678-4634202147232882

Cecchi, N. (2006). Aprendizaje servicio en educación superior. La experiencia latinoamericana. Presentación en el Seminario Internacional Responsabilidad Social Universitaria: Aprendizaje Servicio. Caracas.

Dale, R. (2007). Los efectos de la globalización en la política nacional: Un análisis de los mecanismos. En X. Bonal, A.

Una experiencia transdisciplinar de trabajo comunitario en contextos de formación profesional $\bullet 29$ Samuel Pérez Norambuena, Cristóbal Bravo Ferretti, Marcela Mora Donoso. DIDAC 78 (2021): 20-31 
Tarabini-Castellani \& A. Verger (Comps.), Educación y globalización. Textos fundamentales (pp. 87-114). Buenos Aires: Miño y Dávila.

Escobar, A. (2006). Ciudadanía activa y construcción democrática. En J. León, A. Escobar \& L. Cea (Eds.), Discursos y prácticas de ciudadanía. Debates desde la Región del Bio-Bio (pp. 29-36). Concepción: Ediciones UBB.

Espinoza, O. (2014). Análisis crítico del discurso de las competencias en la formación inicial docente en Chile. Estudios Pedagógicos, XL(2), 147-159.

Espinoza, O. (2016). Neoliberalismo y educación superior en Chile: Una mirada Crítica al rol desempeńado por el Banco Mundial y los "Chicago Boys". Laplage em Revista, 3(3), 93-114.

Green, A. (2007). Educación, globalización y el papel de la investigación comparada. En X. Bonal, A. Tarabini-Castellani \& A. Verger (Comps.), Educación y globalización. Textos fundamentales (pp. 61-86). Buenos Aires: Miño y Dávila.

Freire, P. (1989). La educación como práctica de la libertad. Madrid: Siglo XXI Editores.

Friz Echeverría, C. (2018) Neoliberalismo, educación y ciudadanía en Chile: ¡ciudadanos y/o agentes económicos? En C. Berríos \& C. García (Eds.), Ciudadanias en conflicto. Enfoques, experiencias y propuestas (pp. 155-179). Santiago: Ariadna Ediciones.

Furco, A. \& Billig, S. (2002). Service Learning: The Essence of Pedagogy. Connecticut: IAP.

Iriarte, A. \& Ferrazzino, A. (2013). La cuestión de la formación ciudadana universitaria en Latinoamérica. Construcción y ejercicio de una ciudadanía social. X Jornadas de Sociología. Facultad de Ciencias Sociales, Universidad de Buenos Aires, Buenos Aires.

Leite, D. \& Genro, M. (2012). Avaliação e internacionalização da Educação Superior. Avaliação. Revista da Avaliação da Educação Superior. Campinas, 17(3), 763-785.

López, S. \& Flores C. (2006). Las reformas educativas neoliberales en Latinoamérica. Revista Electrónica de Investigación Educativa, 8(1), 1-15.

Martínez, M. (2003). Transdisciplinariedad, un enfoque para la complejidad del mundo. Revista Visión Docente Con-Ciencia, 1. Recuperado de http://www.concienciactiva.org

Merino, L. (2004). El sistema universitario y la vinculación cultural con el medio. Calidad en la Educación, (20), 77-84. Recuperado de https://doi.org/10.31619/caledu.n20.346

Ministerio de Educación (2018). Orientaciones para la Elaboración del Plan de Formación Ciudadana en Educación Parvularia. Santiago, Chile: Mineduc.

Mollis, M. (2010). Las transformaciones de la Educación Superior en América Latina: Identidades en construcción. Revista Educación Superior y Sociedad, 15(1), 11-24.

Montero, M. (2004). Introducción a la psicología comunitaria. Buenos Aires: Paidós

Morin, E. (2003). Introducción al pensamiento complejo. Barcelona, Gedisa.

Muñoz, C., Martínez, R., Muñoz, C., Orellana, C. \& Salazar, R. (2019). La escuela como espacio de formación ciudada- na. En C. Orellana, R. Salazar \& V. Hasse (Eds.), Formación ciudadana. Santiago: Ril.

Nicolescu, B. (1996). Physique quantique et niveaux de Réalité. En La Transdisciplinarité. Mónaco: Du Rocher.

Oliva, I., Molina, W., Quintero, J. \& Díaz, N. (2018). Prospectiva transdisciplinaria y organización del conocimiento: Una aproximación epistémica a la educación superior en la Región de Magallanes, Chile. Magallania (Punta Arenas), 46(2), 129-142. Recuperado de https://dx.doi.org/10.4067/S0718-22442018000200129

Organización de las Naciones Unidas para la Educación, la Ciencia y la Cultura (Unesco). (1996). Educación Superior para el Siglo xxI. Conferencia Regional sobre Políticas y Estrategias para la Transformación de la Educación Superior en América Latina y el Caribe, Ciudad de La Habana, Cuba. Recuperado de http://www.unesco.org/education/ educprog/wche/declaration_spa.htm

Organización para la Cooperación y el Desarrollo Económicos. (2009). La educación superior en Chile. Revisión de políticas nacionales de educación. Santiago de Chile: Mineduc.

Oyarce, N., Leihy, P. \& Zegers, J. (2020). Crisis de la educación superior en el Chile neoliberal: mercado y burocracia. Educar em Revista, 36. Recuperado de https://dx.doi.org/10.1590/0104-4060.77536

Programa de Naciones Unidades (PNUD) (2004). La democracia en América Latina. Hacia una democracia de ciudadanos. Buenos Aires: Aguilar-Altea-Taurus-Alfaguara.

Programa de Naciones Unidades (PNUD) (2021). La Unesco y los objetivos de desarrollo sostenible. Recuperado de https://es.unesco.org/sdgs

Quintana, I., Mendoza, R., Bravo, C. \& Mora, M. (2018). Enfoque psicosocial. Concepto y aplicabilidad en la formación profesional de estudiantes de Psicología. Revista Reflexión e Investigación Educacional, 2(1), 89-98.

Ramos, Y. \& Parra, A. (2017). Programa "Ser pilo paga" en el marco de la educación superior en Colombia. Diálogos sobre Educación, 14(8), 1-18.

Salazar, G. (2006). Ciudadanía en Chile: antecedentes históricos y perspectivas. En J. León, A. Escobar \& L. Cea (Eds.), Discursos y prácticas de ciudadanía. Debates desde la región del Bio-Bio (pp. 185-197). Concepción: Universidad del Bío-Bío.

Santos, B. (2005). La universidad en el siglo XXI. Para una reforma democrática y emancipadora de la universidad. México: CEIICH/UnAM.

Service-Learning 2000 Center (1996). Service-Learning Quadrants. California: Stanford University.

Schulz, W., Carstens, R., Losito, B. \& Fraillon, J. (Eds.). (2016). International Civic and Citizenship Education Study 2016 Technical Report. Wellington: IEA.

Skewes, J. (2013). La transdisciplina en la formación universitaria. Contenido, Arte, Cultura y Ciencias Sociales, 3, 1-17.

Tapia, M. (2010). La propuesta pedagógica del "aprendizajeservicio": una perspectiva latinoamericana. TzhoeCoen, 23-44.

30 - Una experiencia transdisciplinar de trabajo comunitario en contextos de formación profesional Samuel Pérez Norambuena, Cristóbal Bravo Ferretti, Marcela Mora Donoso. DIDAC 78 (2021): 20-31 


\section{SEmblanzas}

Samuel Pérez Norambuena. Académico del Departamento de Ciencias de la Educación de la

Facultad de Educación y Humanidades en la Universidad del Bío-Bío (Uвв), Chile.

Formador de profesores desde una mirada sistémica, dialógica y relacional en las escuelas de Pedagogía en Educación Física y Educación Básica en la misma casa de estudios. Integrante del grupo de investigación RIADIs. Sus principales líneas de investigación son la formación inicial docente (FID), la formación valórica en la FID, la docencia en educación superior, educación y sociedad.

Cristóbal Bravo Ferretti. Académico del Departamento de Ciencias Sociales de la uBb. Se desempeña como docente en la Escuela de Psicología en la misma casa de estudios. Sus principales líneas de investigación son psicología comunitaria, psicología ambiental, espacio y lugar, etnografía y conflictos socioambientales.

Marcela Mora Donoso. Profesora de Historia y Geografía, magíster en Educación y doctora en Educación. Coordinadora pedagógica de la Unidad de Gestión Curricular y Monitoreo, además se desempeña como docente en el departamento de Ciencias de la Educación de la Facultad de Educación y Humanidades. Docente relatora de cursos para el sistema escolar, desde formación continua UBB, integrante del Grupo de Investigación Profesorado, Políticas de Formación y Praxis Profesional (profor). Sus principales líneas de investigación son los procesos de formación inicial desde una perspectiva crítica transformadora, evaluación emancipadora y currículum.

\footnotetext{
${ }^{1}$ La Universidad del Bío-Bío define la extensión universitaria como "aquella función propia de la educación superior, indeclinable y de servicio público, que tiene como propósito divulgar los logros alcanzados por la institución como resultado de su vinculación e intervención en los procesos de desarrollo social, contribuyendo así a la consolidación de la misión y visión institucional. Asimismo, fomenta actividades complementarias en lo cultural, deportivo, artístico y recreativo con el propósito de aportar soluciones de desarrollo social y potenciar el talento humano". Recuperado de la página web de la Universidad del Bío-Bío.
} 